\title{
Novos registros de Phylloderma stenops Peters na Mata Atlântica, Brasil (Chiroptera, Phyllostomidae)
}

\author{
Carlos E. L. Esbérard ${ }^{1}$ \& Deborah Faria ${ }^{2}$
}

Biota Neotropica v6 (n2)-http://www.biotaneotropica.org.br/v6n2/pt/abstract?short-communication+bn02506022006

Recebido em 06/12/05

Versão Reformulada recebida em 24/04

Publicado em 22/05/05

\begin{abstract}
${ }^{1}$ Departamento de Ecologia, Universidade do Estado do Rio de Janeiro, Rua São Francisco Xavier 540, 20559-900 Rio de Janeiro, RJ, Brasil (cesberard@terra.com.br)

22Departamento de Ciências Biológicas, Universidade Estadual de Santa Cruz,Rodovia Ilhéus-Itabuna km 16, 45650-000

Ilhéus, Bahia, Brazil (deborah@uesc.br)
\end{abstract}

\begin{abstract}
Esbérard, C.E.L. and Faria, D. New records of Phylloderma stenops Peters, 1865 in the Atlantic Forest, Brazil (Chiroptera, Phyllostomidae). Biota Neotrop. May/Aug 2006 vol. 6 no. 2, http://www.biotaneotropica.org.br/v6n2/pt/abstract?shortcommunication+bn02506022006. ISSN 1676-0603
\end{abstract}

The capture of seven bats Phylloderma stenops in three different localities of the Atlantic Forest: Reserva Biológica de Una, Ilhéus (BA), Morro de São João, Casimiro of Abreu (RJ) and in the Estação Ecológica Estadual Paraíso, Guapimirim $(\mathrm{RJ})$, is described.

Key words: occurrence, distribution, biology, rarity

\section{Resumo}

Esbérard, C.E.L. and Faria, D. Novos registros de Phylloderma stenops Peters na Mata Atlântica, Brasil, (Chiroptera, Phyllostomidae). Biota Neotrop. May/Aug 2006 vol. 6 no. 2, http://www.biotaneotropica.org.br/v6n2/pt/abstract?shortcommunication+bn02506022006. ISSN 1676-0603

É relatada a captura de sete exemplares de Phylloderma stenops Peters, 1865 em três diferentes localidades na Mata Atlântica: Reserva Biológica de Una, Ilhéus (BA), Morro de São João, Casimiro de Abreu (RJ) e na Estação Ecológica Estadual Paraíso, Guapimirim (RJ).

Palavras-chave: ocorrência, distribuição, biologia, raridade 


\section{Introdução}

A espécie Phylloderma stenops Peters, 1865 ocorre do México ao sudeste do Brasil (Koopman 1993). No Brasil, existem registros no bioma da Floresta Amazônica para os estados da Amazônia (Bernard 1997 e 2001) e Pará (Jeanne 1970). Guerra (1980) e Souza et al. (2004) citaram a espécie para o estado de Pernambuco, nordeste do Brasil, em bioma de Mata Atlântica. Trajano (1982) relatou a captura desta espécie em ambiente cárstico na Mata Atlântica, em São Paulo, sudeste do Brasil. Segundo Eisenberg \& Redford (1999), não há registros de $P$. stenops em florestas decíduas e secas do Brasil, no entanto, sua ocorrência foi relatada em bioma de Cerrado em Minas Gerais (Trajano \& Gimenez 1998), no Distrito Federal (Bredt et al., 2002) e em Mambaí, Goiás (Esbérard et al. 2005). Esta espécie já foi capturada em vários tipos de habitats, incluindo florestas, áreas abertas e pastagens (Handley 1976) e Gardner (1976) coletou um exemplar a $2900 \mathrm{~m}$ de altitude no norte da América do Sul.

Phylloderma stenops é espécie onívora, ingerindo frutos (família Anonaceae, Cucurbitaceae, Mirtaceae, Cecropiaceae, entre outras) e insetos, incluindo vespas coloniais (e.g. Jeanne 1970, Nowak 1995, Eisenberg \& Redford 1999, Giannini \& Kalko 2004).

\section{Material e Métodos}

Os exemplares foram capturadas com redes de neblina armadas em trilhas já existentes no interior de áreas florestadas e junto a árvores em frutificação. Cada exemplar teve seu peso mensurado com balança de precisão $(0,01 \mathrm{~g})$ e o antebraço medido com paquímetro eletrônico (0,01 mm). Após esta análise os morcegos foram marcados com coleiras providas de cilindros coloridos e soltos no próprio local, uma a quatro horas após a captura. Os animais tiveram a idade estimada em jovem ou adulto, de acordo com a ossificação das epífises.

Os locais amostrados foram: (a) Estação Ecológica Estadual Paraíso, Guapimirim, RJ (22²9,22’S e 042¹2,15’W, $80 \mathrm{~m}$ de altitude), onde as redes foram armadas no Centro de Primatologia do Rio de Janeiro, na borda da mata e sobre o Rio Paraíso (Esbérard, 2004); (b) Fazenda Reunidas, Morro de São João, Casimiro de Abreu, RJ (22²9,96’ S e 4158,92' W, $15 \mathrm{~m}$ de altitude), sendo as redes armadas junto a um bananal e (3) Reserva Biológica de Una, sul da Bahia (15¹0’ S, 3903’ W), com redes armadas no sub-bosque de uma plantação tradicional de cacau (Theobroma cacao, Sterculiaceae), sombreada por floresta nativa (Faria, 2002) (Figura 1).

\section{Resultados e Discussão}

Neste trabalho, são relatadas três novas localidades de captura para $P$. stenops na Mata Atlântica do leste e sudeste do Brasil (Tabela 1).
Um exemplar macho adulto, com testículos abdominais, foi capturado em clareira junto a riacho próximo a indivíduo de Fruta-do-Conde (Annona sp.) (família Anonaceae) em frutificação na Centro de Primatologia do Rio de Janeiro. A captura ocorreu às 19h29min. Um segundo exemplar, também macho com testículos abdominais, foi capturado no mesmo local, às 19:36 h e apresentou fezes com sementes que não foram identificadas. Neste morcego, foram coletados quatro exemplares de Strebla christinae Wenzel, 1966. Um terceiro exemplar, também macho com testículos abdominais, foi capturado no dia 20/09/2004, às 02:30 h, em redes armadas sobre o mesmo riacho, distantes cerca de $150 \mathrm{~m}$ do ponto anteriormente descrito. Todos os exemplares machos analisados apresentaram no momento da captura as glândulas gulares muito desenvolvidas e secretantes. Nesta localidade, ocorrem 35 espécies de morcegos, incluindo duas outras espécies com hábitos onívoros (classificação seguindo Kalko et al. 1996): Phyllostomus hastatus (Pallas, 1767) e Micronycteris hirsuta (Peters, 1869) (Esbérard 2004).

Duas fêmeas, adultas e reprodutivamente inativas, foram capturadas em borda de floresta secundária adjacente a bananal e próximo a várias rochas na Fazenda Reunidas. Os dois exemplares foram capturados no mesmo local e na mesma rede, com intervalo de 78 minutos. O primeiro exemplar apresentou fezes compostas por fragmentos de insetos. Nos remanescentes florestais desta fazenda capturamos 29 espécies de morcegos, tendo sido constatada a presença de numerosa colônia de Phyllostomus hastatus. Phylloderma stenops não havia sido registrada no local durante os três anos anteriores de amostragem.

No inventário realizado na Reserva Biológica de Una foram capturadas 44 espécies de morcegos (Faria 2002), incluindo dois indivíduos de P. stenops. Os indivíduos eram machos, com testículos não escrotados, e foram capturados logo após o crepúsculo.

Phylloderma stenops prefere cavernas ou grutas para refúgio, onde forma grupos não muito numerosos (Trajano 1982, 1996, Trajano \& Gimenez 1998, Bredt et al. 2002, Esbérard et al. 2005). Esta espécie de morcego foi capturada em localidades com elevada riqueza de espécies [24 a 35 espécies - Trajano (1985), Taddei \& Pedro (1996), Bredt et al. (2002), Esbérard et al. (2005)], sugerindo que ocorra apenas em habitats que sustentam comunidades altamente complexas e estruturadas, com grande diversidade de nichos. Mesmo em florestas primárias é capturada em pequeno número, sugerindo baixa densidade (Bernard 1997 e 2001, Clarke \& Downie 2001).

Material testemunho da espécie está depositado na coleção de referência do Projeto Morcegos Urbanos, na Universidade do Estado do Rio de Janeiro e na coleção de referência da Universidade Estadual de Santa Cruz. 


\section{Agradecimentos}

As coletas foram realizadas sob licença especial para coletas (Processos 1755/89-DF e 4156/95-46 AC-SUPES/DF/ IBAMA). Agradecemos o apoio e a permissão para coletas ao Dr. A. Pissinatti, do Centro de Primatologia do Rio de Janeiro, aos proprietários da Fazenda Reunidas e a administração da Reserva Biológica de Una. A Secretaria de Agricultura e Pesca de Casimiro de Abreu forneceu o apoio necessário para as coletas no Morro São João, e o apoio do IBAMA foi fundamental para viabilizar as coletas na Reserva Biológica de Una. Agradecemos a confirmação da identificação da espécie de morcego aos Dr. A. L. Peracchi e C. O. Handley Jr. e dos ectoparasitas ao Dr. G. Graciolli.

\section{Referências bibliográficas}

BREDT, A., UIEDA, W. \& MAGALHÃES, E.D. 1999. Morcegos cavernícolas da região do Distrito Federal, centro-oeste do Brasil (Mammalia, Chiroptera). Rev. Brasil. Zool. 16(3):731-770.

CLARKE, F.M. \& DOWNIE, J.R. 2001. A bat (Chiroptera) survey of Mora rainforest in Trinidad's Victoria -Mayaro Forest Reserv. Biod. Conserv. 10:725-736.

EISENBERG, J.F.\& REDFORD, K.H. 1999. Mammals of the neotropics. The Northern Neotropics, The Central Neotropics: Ecuador, Peru, Bolivia, Brazil. University of Chicago Press, Chicago.

ESBÉRARD, C.E.L. 2004. Novo registro de Micronycteris hirsuta (Peters) (Mammalia, Chiroptera) na Mata Atlântica, Estado do Rio de Janeiro, Brasil. Rev. Brasil. Zool. 21:403-404.

ESBÉRARD, C.E.L.; MOTA, J.A. \& PERIGRO, C. 2005. Morcegos cavernícolas da APA Nascentes do Rio Vermelho, Goiás. Rev. Brasil. Zooc. 7: 311-325.

FARIA, D. 2002. Comunidade de morcegos em uma paisagem fragmentada da Mata Atlântica do sul da Bahia, Brasil. Tese de doutorado, Universidade Estadual de Campinas, São Paulo.

GARDNER, A. L. 1976. The distributional status of some Peruvian Mammals. Occ. Pap. Mus. Zool. 4:1-18.

GIANNINI, N.P. \& KALKO. E.K.V. 2004. Trophic structure in a large assemblage of phyllostomid bats in Panama. Oikos 105:209-220.

GUERRA, D.O. 1980. Registro adicional de Phylloderma stenops Peters, 1865 (Chiroptera-Phyllostomatidae) para o Brasil. Rev. Nordestina Biol. 3 (supl.):141-143.

HANDLEY, C.O. JR. 1976. Mammals of the Smithsonian Venezuelan Project. Brigham Young Univ. Sci. Bull., Biol. Ser. 20:1-89.

JEANNE R. L. 1970. Note on a bat (Phylloderma stenops) preying upon the brood of a social wasp. J. Mammal. 51:624-625.
KALKO, E.K.V., C.O. HANDLEY JR. \& HANDLEY, D. 1996. Organization, diversity and long-term dynamics of a neotropical bat community. in Long-term studies of vertebrate communities (M.L. Cody \& J.A. Smallwood, eds), Academic Press, New York, p.503-553.

KOOPMAN, K. F. 1993. Chiroptera. in Mammalian species of the world (Wilson, D. E., and D. M. Reeder, eds). Smithsonian Institution Press, Washington, p.137-241.

NOWAK, R.M. 1995. Walker's mammals of the world. The John Hopkins University Press, $4^{\text {nd }}$ ed, Baltimore.

PULCHÉRIO-LEITE, A. P., MENEGHELLI, M. \& TADDEI, V.A. 1998. Morcegos (Chiroptera: Mammalia) dos Pantanais de Aquidauana e da Nhecolândia, Mato Grosso do Sul. I. Diversidade de espécies. Ensaios em Ciências 2:149-163.

SOUSA, M.A.N., LANGGUTH, A. \& GIMENEZ, E.A. 2004. Mamíferos dos brejos de altitude Paraíba e Pernambuco. In Brejos de altitude em Pernambuco e Paraíba - História natural, ecologia e conservação (K.C. Porto, J.J.P. Cabral \& M. Tabareli, eds). Série Biodiversidade 9. Ministério do Meio Ambiente, Brasília, p.229-254.

TADDEI, V. A. \& PEDRO, W. 1996. Morcegos (Chiroptera: Mammalia) do Vale do Ribeira, Estado de São Paulo: diversidade de espécies. In Anais do VIII Seminário Regional de Ecologia (J.E. Santos, ed.). Programa de PósGraduação Ecologia e Recursos Naturais, Universidade Federal de São Carlos, São Carlos, p.911-919.

TRAJANO, E. 1982. New records of bats from southeastern Brazil. J. Mammal. 63: 529.

TRAJANO, E. 1985. Ecologia de populações de morcegos cavernícolas em uma região cárstica do sudeste do Brasil. Rev. Brasil. Zool. 2:255-320.

TRAJANO, E. 1996. Protecting caves for the bats or bats for the caves? Chiroptera Neotropical 1:19-22.

TRAJANO, E. \& GIMENEZ, E.A. 1998. Bat community in a cave from eastern Brazil, including a new record of Lionycteris (Phyllostomidae, Glossophaginae). Stud. Neot. Fauna Environ. 33:69-75.

Título: Novos registros de Phylloderma stenops Peters na Mata Atlântica, Brasil, (Chiroptera, Phyllostomidae).

Autores: Esbérard, C.E.L. \& Faria, D.

Biota Neotropica, Vol. 6 ( número 2): 2006

http://www.biotaneotropica.org.br/v6n2/pt/ abstract?short-communication+bn02506022006

Recebido em 06/12/05 - Versão Reformulada recebida em 24/04 - Publicado em 22/05/05

ISSN 1676-0603 


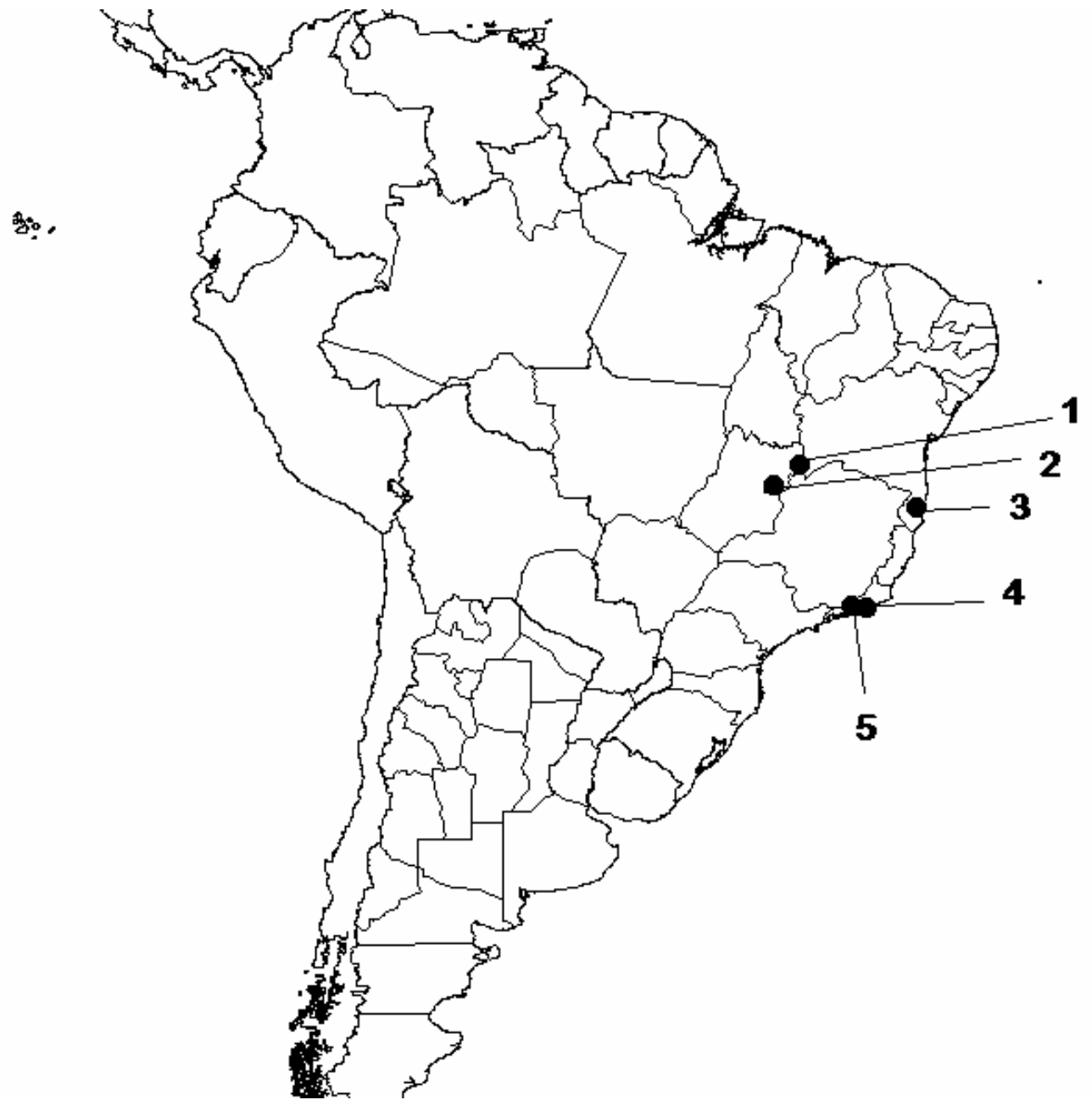

Figura 1. Ocorrência de Phylloderma stenops. (1) APA Nascentes do Rio Vermelho, Mambaí (Esbérard et al. 2005), (2)Goiás; Distrito Federal, DF (Bredt et al. 1999); (3) Reserva de Una, Ilhéus, Bahia; (4) Morro de São João, Rio de Janeiro; (5) Estação Ecológica Estadual Paraíso, Rio de Janeiro.

Figure 1. Occurrence of Phylloderma stenops. (1) APA Nascentes do Rio Vermelho, Mambaí (Esbérard et al. 2005), (2)Goiás; Distrito Federal, DF (Bredt et al. 1999); (3) Reserva de Una, Ilhéus, Bahia; (4) Morro de São João, Rio de Janeiro; (5) Estação Ecológica Estadual Paraíso, Rio de Janeiro. 
Tabela 1. Localidades, data das capturas e medidas de Phylloderma stenops

Table 1. Localities, dates of capture and measures of Phylloderma stenops.

\begin{tabular}{|c|c|c|c|c|c|}
\hline \multicolumn{2}{|l|}{ LOCALIDADES } & \multirow{2}{*}{$\begin{array}{l}\text { DATA } \\
07 / 11 / 97\end{array}$} & \multirow{2}{*}{$\begin{array}{l}\text { SEXO } \\
\text { macho }\end{array}$} & \multirow{2}{*}{$\begin{array}{c}\begin{array}{c}\text { ANTEBRAÇO } \\
\text { (mm) }\end{array} \\
78,00\end{array}$} & \multirow{2}{*}{$\begin{array}{c}\text { PESO (g) } \\
87,0\end{array}$} \\
\hline $\begin{array}{l}\text { Estação Ecológica } \\
\text { Guapimirim (RJ) }\end{array}$ & Paraíso, & & & & \\
\hline $\begin{array}{l}\text { Estação Ecológica } \\
\text { Guapimirim (RJ) }\end{array}$ & Paraíso, & $21 / 03 / 98$ & macho & 77,55 & 80,0 \\
\hline \multicolumn{2}{|c|}{ Guapimirim (RJ) } & $20 / 09 / 04$ & macho & 77,52 & 76,2 \\
\hline \multicolumn{2}{|c|}{$\begin{array}{l}\text { Fazendas Reunidas, Morro de São } \\
\text { João, Casimiro de Abreu (RJ) }\end{array}$} & $05 / 11 / 01$ & fêmea & 78,70 & 89,9 \\
\hline \multicolumn{2}{|c|}{$\begin{array}{l}\text { Fazendas Reunidas, Morro de São } \\
\text { João, Casimiro de Abreu (RJ) }\end{array}$} & $05 / 11 / 01$ & fêmea & 75,00 & 87,2 \\
\hline \multicolumn{2}{|c|}{$\begin{array}{l}\text { Reserva Biológica de Una, Ilhéus } \\
\text { (BA) }\end{array}$} & 29/11/98 & macho & 73,30 & 57,0 \\
\hline \multicolumn{2}{|c|}{$\begin{array}{l}\text { Reserva Biológica de Una, Ilhéus } \\
\text { (BA) }\end{array}$} & 29/11/98 & macho & - & 68,0 \\
\hline
\end{tabular}

http://www.biotaneotropica.org.br 